\title{
Numerical analysis of the hydrodynamic behaviour of immiscible metallic alloys in twin-screw rheomixing process
}

\author{
H. Tang ${ }^{1,2}$, L.C. Wrobel ${ }^{2}$, Z. Fan ${ }^{1}$ \\ ${ }^{1}$ Brunel Centre for Advanced Solidification Technology, Brunel University, Uxbridge UB8 3PH, UK \\ ${ }^{2}$ School of Engineering and Design, Brunel University, Uxbridge UB8 3PH, UK
}

\begin{abstract}
A numerical analysis by a VOF method is presented for studying the hydrodynamic mechanisms of the rheomixing process by a twin-screw extruder (TSE). The simplified flow field is established based on a systematic analysis of flow features of immiscible alloys in TSE rheomixing process. The studies focus on the fundamental microstructure mechanisms of rheological behaviour in shear-induced turbulent flows. It is noted that the microstructure of immiscible alloys in the mixing process is strongly influenced by the interaction between droplets, which is controlled by shearing forces, viscosity ratio, turbulence, and shearing time. The numerical results show a good qualitative agreement with the experimental results, and are useful for further optimisation design of prototypical rheomixing processes.
\end{abstract}

Key words: immiscible alloys, rheomixing process, VOF, shear-induced turbulent flow, twin-screw extruder, optimisation

\section{Introduction}

Immiscible alloys, such as $\mathrm{Al}-\mathrm{Pb}$ and $\mathrm{Al}-\mathrm{Bi}$, are advanced materials used for bearing applications in the automotive industry. For instance, in a sliding bearing, a soft $\mathrm{Pb}$ phase well dispersed in a solid matrix will produce characteristics of low friction and good wear resistance. The application of these alloys, however, has been limited, due to metallurgical problems related to liquid phase separation during casting. The components of immiscible alloys are only completely miscible above the temperature $T_{c}$ indicated by the binodal line in Fig. 1. When a single-phase liquid is cooled below the temperature $T_{c}$ shown in Fig. 1, it transforms into two liquids. Generally, these droplets may settle due to gravity, temperature or concentration gradients. Normally, large compositional and density differences invariably exist between the two liquid phases, leading to a rapid spatial phase separation or macrosegregation during solidification on earth. The microstructure of such casting is a complex interplay of nucleation, growth, Stokes and Marangoni motion, the interaction of the individual flow fields around the moving droplets and their interaction with the solidification. Evidences show that the solidified microstructure of cast immiscible alloys strongly depends on the hydrodynamic behaviour of the liquids during cooling [1]. 


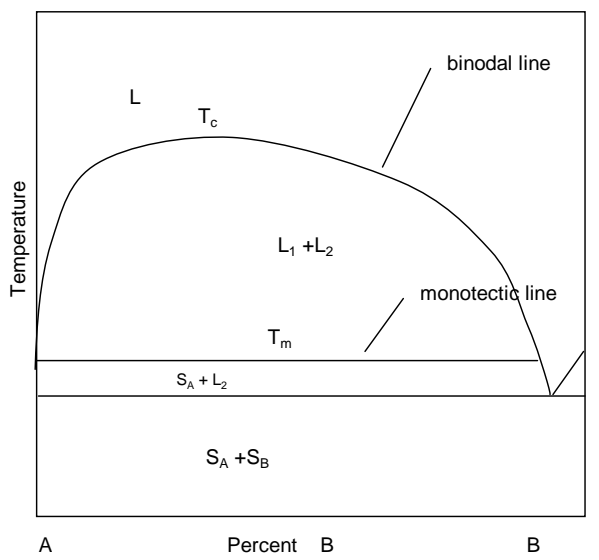

Fig. 1 Scheme of a typical binary phase diagram with a liquid miscible gap.

Research shows that a fine dispersion of minority phase particles in a hypermonotectic alloy can be obtained by rapid quenching or fast cooling, thus passing through the miscibility gap within a second or less. For example, a special planar flow casting process in the horizontal direction and a vertical strip casting process were invented using the fast cooling method [2]. However, results show that the soft phase drops concentrate in the middle of the strip due to Marangoni motion. A novel TSE rheomixing process has been successfully developed in our laboratory for casting immiscible alloys [3]. Here, we present a numerical analysis of the hydrodynamic behaviour of an immiscible metallic drop in a shearinduced turbulent flow, which is the main flow feature in the TSE rheomixing process. The emphasis of the present work is to investigate hydrodynamic behaviours and to draw qualitative differences in the features produced by different shearing approaches and properties.

\section{Analysis of the twin-screw rheomixing processing}

A rheomixing process was developed based on previous experience in the processing of semisolid metal (SSM) slurry by a twin-screw extruder (TSE) [4]. TSE is commonly used in polymer processing $[5,6]$, and provides sufficient shear flow in the rheomixing process to create a fine and homogeneous droplet dispersion, and enough viscous force to counterbalance the Stokes and Marangoni motions by choosing the proper operating temperature. The intermeshing co-rotating twin-screw extruder is used for mixing immiscible alloys. Experimental results show that fine and spherical $\mathrm{Pb}$ particles are distributed uniformly in the Zn matrix phase [3]. 
The flow field of the intermeshing co-rotating twin-screw extruder undergoes cyclic stretching, folding, and reorienting [7]. The flow patterns inside a TSE are complex, as shown in fig. 7 of [7], and studies have confirmed that the fluid moves in figure " 8 " motions around the periphery of the screws $[8,9]$. Experimental velocity fields inside a TSE were reported in [10]. A global view of the laminar flow within a twin-screw is illustrated in [11], highlighting the stretching process takeover of material due to intensive shear flow [12]. The maximum shear rate is produced by the intermeshing regions in minimum gaps located between the screw and barrel, and tip to tip of the screws. Although detailed studies of twin-screw extruders for polymer processing have been performed numerically [13-17] and experimentally [18,19], their results are still limited for a proper understanding of the microscopic principles of immiscible liquid alloys due to the complex multiphase characteristics both in the hydrodynamic and metallurgical aspects.

Basically, the main feature of a twin-screw extruder is a strong shear flow field produced by corotating intermeshing screws $[5,11,20]$. The droplets are created in the microscopic scale, and the turbulent flow enhanced by mixing, swirling and pumping actions in a macroscopic scale. The rheomixing process is shown in Fig. 2. Two immiscible liquid alloys are stirred before pouring into a twin-screw extruder, and the minority phase $\mathrm{Pb}$ liquid is in the form of larger droplets. The main concern for the rheomixing process is droplet interaction, including rupturing, coalescence and suspension, which affects the microstructure of immiscible alloy castings.

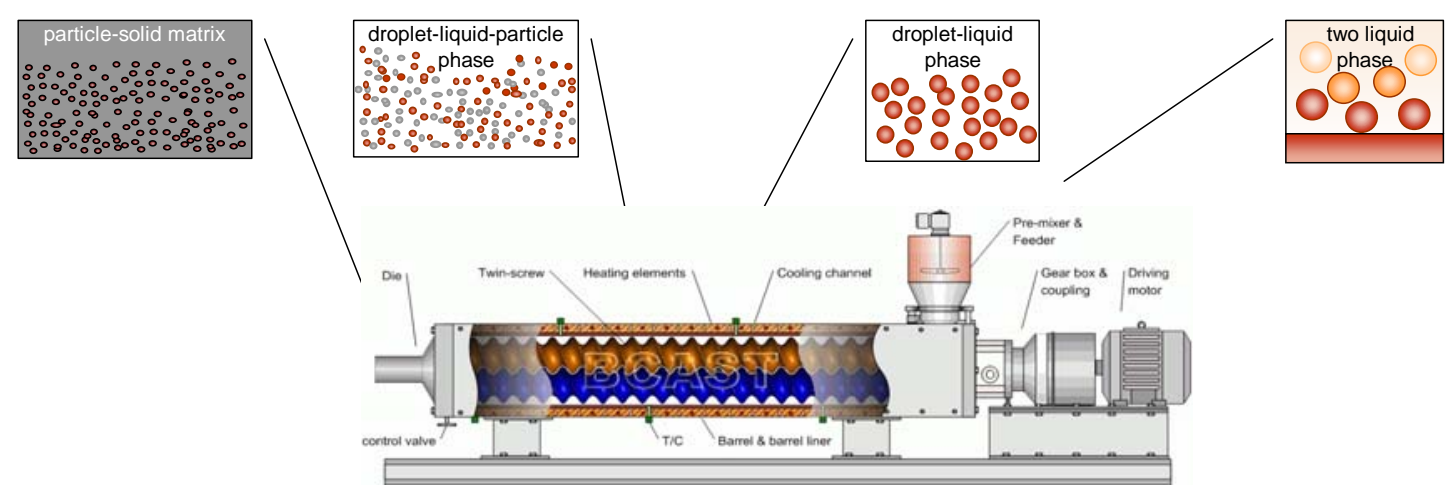

Fig. 2 Schematic illustration of the twin-screw rheomixing process. 
The procedures for rheomixing can be described in four stages, according to rheological behaviour:

1. Formation of $\mathrm{Pb}$ drop in $\mathrm{Zn}$ matrix liquid phase: $\mathrm{Pb}$ drops formed from two liquid phases by shear stress, induced by the action of mechanical devices; the minority phase is in the form of large drops.

2. Rupture and coalescence of $\mathrm{Pb}$ drop: $\mathrm{Pb}$ drops breakup into small droplets by a twin-screw under high speed.

3. $\mathrm{Pb}$ liquid droplet and $\mathrm{Zn}$ solid particle collision: collisions occur when the temperature is below $\mathrm{T}_{\mathrm{m}}$, the $\mathrm{Pb}$ drop is broken up into fine droplets by collision with $\mathrm{Zn}$ solid particles in turbulence flow activated mainly by mixing block elements, to prevent the coarsening of $\mathrm{Zn}$ particles.

4. Stability of dispersion and suspension: $\mathrm{Pb}$ droplets are dispersed by turbulence and suspended in the $\mathrm{Zn}$ matrix phase due to the increased viscosity of the $\mathrm{Zn}$ matrix phase in semisolid state. This prevents $\mathrm{Pb}$ droplets from further coagulation.

Drop deformation and breakup in pure shear flow have been studied since 1932 [21,22]. More recently, experimental and computational observations of drop formation and breakup have been reported [23-27]. A numerical study of immiscible drops in turbulent flow shows that the drop shape depends on the local flow regime, classified by four turbulence structure types [28]. A comprehensive review of immiscible fluid mixing was given in [29], which focused on stages from steady to unsteady features.

Drop deformation in steady state can be measured by the Taylor deformation parameter $D=(L-B) /$ $(L+B)$, where $B=r_{d}(1-D)$ is the minor axis of the drop, $L=r_{d}(1+D)$ is the major one, and $r_{d}$ is the drop radius. For drop deformation in unsteady state, the drop was elongated under various shapes depending on the imposed stress, and finally starts breaking up. After a fully broken up, refinement and coalescence are occurred by the interactions between daughter droplets. However, the mixing behaviour of daughter droplets in shear-induced flow has not been reported in the literature. Moreover, the hydrodynamic behaviour of immiscible liquid alloys in a shear-induced mixing process has not been examined, especially in a twin-screw extruder. Here, the essential micro-mechanism of immiscible liquid alloys in rheomixing is presented. The numerical simulations are conducted through the volume-of-fluid (VOF) method [30] with piecewise linear interface construction (PLIC) scheme [31], algebraic multigrid (AMG) approach [32] and $k-\varepsilon$ turbulence model [33]. The rupturing, interaction and dispersion of droplets, the essential microscopic mechanisms of the twin-screw extruder, are investigated to improve further our understanding of the rheomixing process. The 
numerical methods used in these investigations have been previously validated [34]. The studies reveal a wealth of interesting rheological and microstructural features that provide qualitative insights into rheomixing, which are consistent with previous experimental work.

\section{Flow configuration}

The simplified flow fields for studying the main microstructural rheomixing mechanisms in a twinscrew extruder are illustrated in Fig. 3. The imposed shear rate $\dot{\gamma}$ is given by the following equation [35]:

$$
\dot{\gamma}=2 n \pi\left(r_{s} / \delta-1\right)
$$

where $r_{s}$ is the screw radius, $n$ is the screw rotational speed and $\delta$ corresponds to the gap or channel depth of the twin-screw extruder.
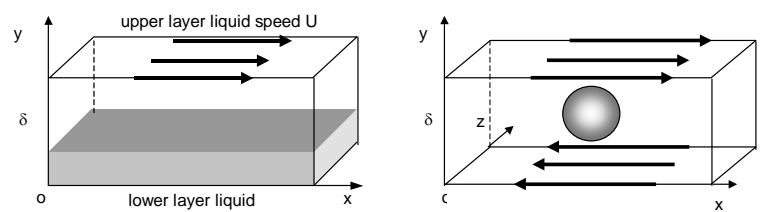

Fig. 3 Schematics of the computational domain in shear-induced flow for studying the phenomena of drop formation (left) and drop rupturing (right); spatially periodic in the $\mathrm{x}$ and $\mathrm{z}$ directions.

Numerical simulations are built on two computational domains. The first is for studying two-phase mixing. The immiscible $\mathrm{Zn}-\mathrm{Pb}$ binary alloy system is initially stratified, and a shear rate is imposed on the upper and lower layers. The second computational domain is for studying drop breakup and refinement. $\mathrm{A} \mathrm{Pb}$ drop is initially suspended in the matrix phase, and assumed to have an undeformed radius $r_{d}$ and viscosity $\mu_{d}$, while the matrix phase has viscosity $\mu_{m}$, and the distance between barrel and screw is $\delta$. Computations are conducted as two-dimensional due to the limitations of computer capabilities. The two-dimensional domain size is $16 \times 4$, grid size is $128 \times 32$.

Model experiments of parallel disks were performed in order to study the fundamental mechanism of immiscible polymeric materials in a twin-screw extruder [36]. However, numerical simulations have advantages since various shear rate profiles can be established for setting up initial and boundary 
conditions, and more complex forces can be easily imposed to reflect the special operating condition and screw configuration.

There are four dimensionless parameters in our numerical simulations: the capillary number $\mathrm{Ca}=\dot{\gamma} \mu_{m}$ $r_{d} / \sigma$, where $\sigma$ is the interfacial tension and the average shear rate is defined as $\dot{\gamma}=U / \delta$; the Weber number $\mathrm{Web}_{\text {drop }}=\operatorname{Re} \mathrm{Ca}=\rho r_{d} U^{2} / \sigma$; the viscosity ratio $\lambda=\mu_{d} / \mu_{m}$; and the Reynolds number $\operatorname{Re}_{\text {drop }}=\rho_{m} \dot{\gamma} r_{d}^{2} / \mu_{m}$, where subscript ${ }_{\mathrm{m}}$ is for matrix phase and $\mathrm{d}_{\mathrm{d}}$ is for drop phase.

In the simple shear flow, the drop hydrodynamics is mainly influenced by two competing forces. One is the viscous shear stress $\mu_{m} \dot{\gamma}$ of the matrix phase, which causes drop deformation, and the other is the characteristic Laplace pressure $\sigma / r_{d}$, which resists the deformation. The capillary number $\mathrm{Ca}$, defined as the ratio between these two forces, provides a useful measure of the efficiency of the shear flow to deform the drop. The viscosity ratio is defined as the ratio of drop viscosity to matrix phase viscosity. The Reynolds number Re is the ratio of inertia force to viscous force.

The twin-screw used in the rheomixing process has a $16 \mathrm{~mm}$ diameter at tip and $3 \mathrm{~mm}$ groove with a special profile [37] to achieve high shear rate and enhance the positive displacement pumping action. The maximum rotation speed of the screw is designed at $1000 \mathrm{rpm}$, which corresponds to a shear rate at $4082 \mathrm{~s}^{-1}$ in the gap between the tip of the screw flight and the barrel. The rotation speed for the experimental work in our laboratory was set at $800 \mathrm{rpm}$. The status of the immiscible alloy liquid during the twin-screw processing is controlled by a temperature control system which ensures a proper viscosity of the matrix phase from start to end [3].

The thermophysical properties of immiscible metallic $\mathrm{Zn}-\mathrm{Pb}$ binary alloys are taken from [38], while phase equilibrium data are taken from [39].

\section{Numerical modelling}

\subsection{Governing equations of interface motion}

Metallic $\mathrm{Zn}-\mathrm{Pb}$ binary alloy are here considered as two-phase immiscible fluid systems in isothermal state, with different density and viscosity between the two phases. The domain of interest contains an unknown free boundary, which undergoes severe deformation and separation.

Several methods are available for tracking the interface motion, such as tracking methods (Lagrangian methods), including moving-mesh, front tracking [40], boundary integral [41] and particle 
schemes; and capturing methods (Eulerian methods), including volume tracking [42-46], volume-offluid (VOF) [30], continuum convection, level set [47] and phase field. Each of these methods has its advantages and disadvantages. The volume-of-fluid (VOF) method provides a simple way of treating some topological changes of the interface, such as merging and folding, that may be difficult to handle accurately and directly by other methods. It seems there are advantages in the use of VOF methods for the simulation of drop behaviour in turbulent shear flow.

In the VOF method, the motion of the interface between two immiscible liquids of different density and viscosity is defined by a volume fraction function $C$, and the interface is tracked by the following three conditions:

$$
\begin{aligned}
& C=0 \text { fluid } 1 \\
& C=1 \text { fluid } 2 \\
& 0<C<1 \text { interface between the two fluids }
\end{aligned}
$$

According to the local value of $C$, appropriate properties and variables are assigned to each control volume within the domain.

The volume fraction function $C$ is governed by the volume fraction equation:

$$
\frac{\partial C}{\partial t}+\mathbf{u} \cdot \nabla C=0
$$

where $\mathbf{u}$ is the flow velocity.

The fluids in this study are subject to the incompressibility condition:

$$
\nabla \cdot \mathbf{u}=0
$$

The flow is governed by a single momentum equation, with the resulting velocity field shared among the phases:

$$
\rho\left(\frac{\partial \mathbf{u}}{\partial t}+\mathbf{u} \cdot \nabla \mathbf{u}\right)=-\nabla p+\mu \nabla^{2} \mathbf{u}+\rho g+F
$$

where $F$ stands for body forces, $g$ for gravity acceleration, and $p$ for pressure.

The properties appearing in the momentum equation are determined by the presence of the component phase in each control volume, that is, the average value of density and viscosity are interpolated by the following formulas

$$
\begin{aligned}
& \rho=C_{\mathrm{m}} \rho_{\mathrm{m}}+\left(1-C_{\mathrm{m}}\right) \rho_{\mathrm{d}} \\
& \mu=\mathrm{C}_{\mathrm{m}} \mu_{\mathrm{m}}+\left(1-C_{\mathrm{m}}\right) \mu_{\mathrm{d}}
\end{aligned}
$$


The VOF approach uses a sheared-field approximation since a single set of mass and momentum equations is adopted. The limitation is that when large velocity differences exist between the phases, the accuracy of the velocities computed near the interface can be adversely affected. A sufficient fine mesh is required in order to prevent the loss of mass. This may be generated adaptively at each time step, but results in increased computing time.

\subsection{Geometric reconstruction scheme for VOF}

The formulation of the VOF model requires that the convection and diffusion fluxes through the control volume faces be computed and balanced with source terms within the cell itself. The interface will be approximately reconstructed in each cell by a proper interpolating formulation, since interface information is lost when the interface is represented by a volume fraction field. The geometric reconstruction PLIC (piecewise linear interface construction) scheme is employed because of its accuracy and applicability for general unstructured meshes, compared to other methods such as the donor-acceptor, Euler explicit, and implicit schemes. A VOF geometric reconstruction scheme is divided into two parts: a reconstruction step and a propagation step. The key part of the reconstruction step is the determination of the orientation of the segment. This is equivalent to the determination of the unit normal vector $\mathbf{n}$ to the segment. Then, the normal vector $\mathbf{n}$ and the volume fraction $\mathrm{C}$ uniquely determine a straight line. Once the interface has been reconstructed, its motion by the underlying flow field must be modelled by a suitable algorithm [31].

\subsection{Implementation of surface tension}

Surface tension along an interface arises as the result of attractive forces between molecules in a fluid. In a droplet surface, the net force is radially inward, and the combined effect of the radial components of forces across the entire spherical surface is to make the surface contract, thereby increasing the pressure on the concave side of the surface. At equilibrium in this situation, the opposing pressure gradient and cohesive forces balance to form spherical drops. Surface tension acts to balance the radially inward inter-molecular attractive force with the radially outward pressure gradient across the surface. Implementation of surface tension was reviewed by Scardovelli and Zaleski [28]. Here, surface tension is applied using the continuum surface force (CSF) scheme of Brackbill et al. [48]. The 
addition of surface tension to the VOF method is modelled by a source term in the momentum equation. The pressure drop across the surface depends upon the surface tension coefficient $\sigma$,

$$
\Delta p=\sigma\left(\frac{1}{R_{1}}+\frac{1}{R_{2}}\right)
$$

where $\mathrm{R}_{1}, \mathrm{R}_{2}$ are the two radii, in orthogonal directions, to measure the surface curvature. In the CSF formulation, the surface curvature is computed from local gradients of the surface normal at the interface. The surface normal $\boldsymbol{n}$ is defined by

$$
\boldsymbol{n}=\nabla C_{d}
$$

where $C_{\mathrm{d}}$ is the secondary phase volume fraction.

The curvature $k$ is defined in terms of the divergence of the unit normal $\hat{n}$ :

$$
k=\nabla \cdot \hat{\mathbf{n}}=\frac{1}{|\boldsymbol{n}|}\left[\left(\frac{\boldsymbol{n}}{|\boldsymbol{n}|} \cdot \nabla\right)|\boldsymbol{n}|-(\nabla \cdot \boldsymbol{n})\right]
$$

where

$$
\hat{\boldsymbol{n}}=\frac{\boldsymbol{n}}{|\boldsymbol{n}|}
$$

The surface tension can be written in terms of the pressure jump across the interface, which is expressed as a volume force added to the momentum equation

$$
F=2 \sigma k C_{d} \nabla C_{d}
$$

The CSF model allows for a more accurate discrete representation of surface tension without topological restrictions, and leads to surface tension forces that induce a minimum in the free surface energy configuration.

\subsection{Numerical approach}

The solution algorithm involves the use of a control-volume-based technique to convert the governing equations to algebraic equations that can be solved numerically. Non-linear governing equations are linearized by an implicit scheme to produce a system of equations for the dependent variable in every computational cell. A point implicit Gauss-Seidel linear equation solver is then used, in conjunction with an algebraic multigrid (AMG) method, to solve the resultant scalar system of equations for the dependent variable in each cell [49].

The governing equation can be discretised in the finite volume method (FVM) as follows: 


$$
\frac{(\rho \phi)_{f}{ }^{t+\Delta t}+(\rho \phi)_{f}{ }^{t}}{\Delta t} \Delta V+\sum_{f}^{N f a c e s} \rho_{f} \phi_{f} V_{f} A_{f}=\sum_{f}^{N f a c e s} \Gamma_{f}(\nabla \phi)_{n} A_{f}+S_{\phi} \Delta V
$$

where $N_{\text {faces }}$ is the number of faces enclosing a cell

$\phi_{f}$ is the value of $\phi$ convected through face $f$

$A_{f}$ is the area of face $f,|A|\left(=\left|A_{x} \hat{i}+A_{y} \hat{j}\right|\right.$ in 2D)

$(\nabla \phi)_{n}$ is the magnitude of $\nabla \phi$ normal to face $f$

$V$ is the cell volume

$\Gamma_{\phi}$ is a diffusion coefficient for $\phi$

$S_{\phi}$ is a source of $\phi$ per unit volume

A linearized form of equation (15) can be written as

$$
a_{p} \phi+\sum_{n b} a_{n b} \phi_{n b}=b
$$

where the subscript $n b$ refers to neighbour cells, and $a_{p}$ and $a_{n b}$ are the linearized coefficients for $\phi$ and $\phi_{n b}$.

The pressure-velocity coupling is achieved by using the pressure-implicit with splitting of operators (PISO) scheme [50]. Since body forces (such as surface tension) are to be considered in the calculation, a special treatment (body-force-weighted scheme) is adopted to deal with the equilibrium of the body force and pressure gradient terms in the momentum equation. This induces an extra correction in the source term of the pressure and face flux rate equations, and the flow generally achieves a realistic pressure field very early in the iterative process. The standard $k-\varepsilon$ turbulence model is employed for turbulence-imposed flows.

The computational domain in our problem requires very refined grids, because of the need to capture the drop formation and breakup at a very fine scale. An efficient solution is provided by the algebraic multigrid approach, which accelerates the convergence of the solver by computing corrections on a series of coarse grid levels when the domain contains a large number of control volumes.

\section{Results and discussion}

5.1 Drop formation for immiscible binary alloy in shear-induced flows 
The simulation results are shown in Fig. 4. In the immiscible $\mathrm{Zn}-\mathrm{Pb}$ binary alloy system, there is a large difference in the density of the two phases. When the temperature is above the bimodal line, the immiscible $\mathrm{Zn}-\mathrm{Pb}$ binary alloy system is in a single phase, a homogeneous liquid mixed in molecular state. If cooled below the bimodal line, it transfers to a two-phase liquid system. The Pb phase sinks to the bottom due to sedimentation by the effect of gravity. The two separated phases can be mixed by imposing an external force such as mechanical stirring. The boundary of the computation domain is periodic in the $\mathrm{x}$-direction and z-direction, and the initial shear rate field has a non-linear profile produced by a stirring action.

The numerical investigations are performed for one-sided shear-induced flows. The simulations ended after the first few droplets were formed due to limitations of our computer capabilities, since each iteration takes more than an hour after the first few droplets are formed.

The results of numerical simulations for one-sided shear-induced flow are shown in Fig. 4. The first droplets were formed around $3.6 \mathrm{~ms}$, and the size of a smallest droplet is quite small. The droplet formation occurred in the sheared layer, the wave of $\mathrm{Pb}$ phase appeared first at the time $2.0 \mathrm{~ms}$, followed by a protruding finger at the time $3.2 \mathrm{~ms}$. The protruding finger then broke up, and droplets formed at the time $3.6 \mathrm{~ms}$.

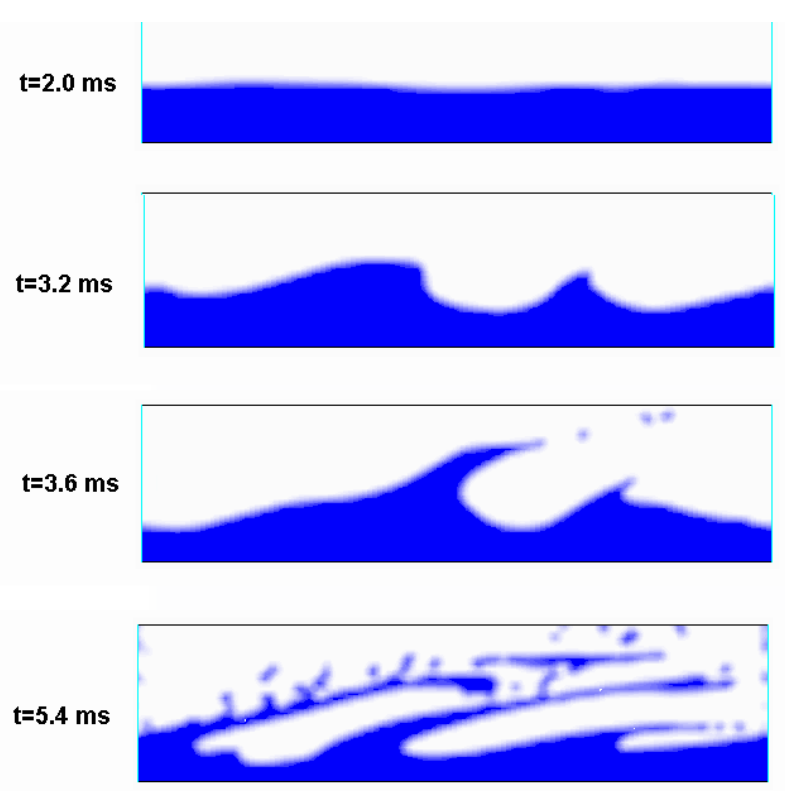

Fig. 4 Numerical evolution of droplet formation in a 2D one-sided shear-induced flow( case1). 
The process of drop formation is mainly controlled by the shear rate, and the minority phase cannot entirely form drops with a lower shear rate even after a long shearing time as shown in Fig. 5.

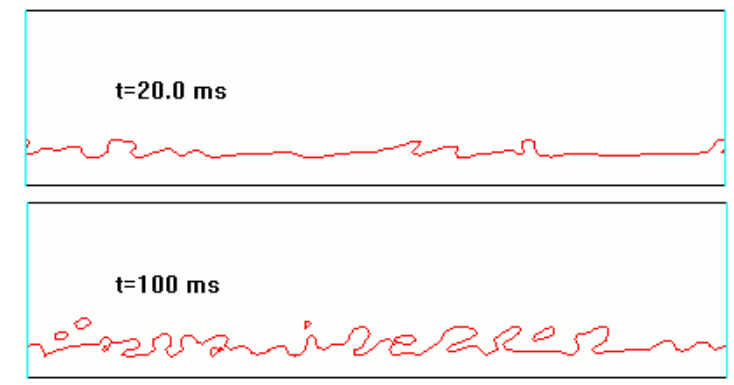

Fig. 5 Numerical evolution of droplet formation in a 2D two-sided shear-induced flow.(case3)

Flows composed of two immiscible liquids and undergoing shearing motion can form fingers as a result of an interfacial instability due primarily to the viscosity jump. The jump is in the tangential velocity gradient across the interface, and can be though of as a viscous counterpart of the KelvinHelmholtz instability [51]. The process of drop formation displays a good agreement with hydrodynamic characteristics observed experimentally [52 54].

\subsection{Effect of initial shear rate in the drop rupturing process}

After drop formation, $\mathrm{Pb}$ metallic drops are broken up by imposed shear forces to create droplets in the channel of the twin-screw. The initial shear rate, as defined in Fig. 3, reflects the basic conditions of flow within TSE and seriously impact on the rupturing behaviour of a $\mathrm{Pb}$ metallic drop in $\mathrm{Zn}$ liquid metal. For $\mathrm{Ca}=3.2, \lambda=1$, rupturing is faster in two-sided shear-induced flow and rapid profile of initial shear rate than one-sided shear-induced flow and linear profile of initial shear rate, as shown in Fig. 6 and Fig. 7.

\subsection{Effect of viscosity variation in the rheomixing process}

The dynamic behaviour of droplets in flows with different viscosity ratio are very different. A viscosity ratio $\lambda=1$ results in a quick breakup and fine droplets. Decreasing the viscosity ratio to $\lambda=0.5$ leads to increasing the size of daughter droplets and a delay in forming the first daughter droplet, but more spherical droplet shapes than in an equal viscosity flows as shown in Fig. 8. 


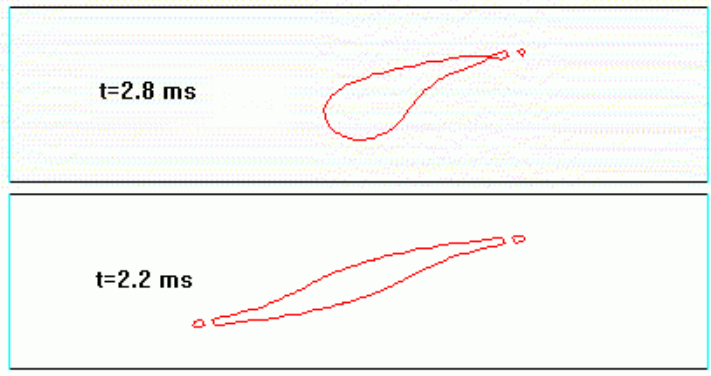

Fig. 6 Numerical evolution of rupturing behaviour of a $\mathrm{Pb}$ metallic drop in shear-induced flow. $\mathrm{Ca}=3.2$, $\lambda=1$, rapid profile of initial shear rate for one-sided shear (upper, case4) and two-sided shear-induced flow (lower, case5).

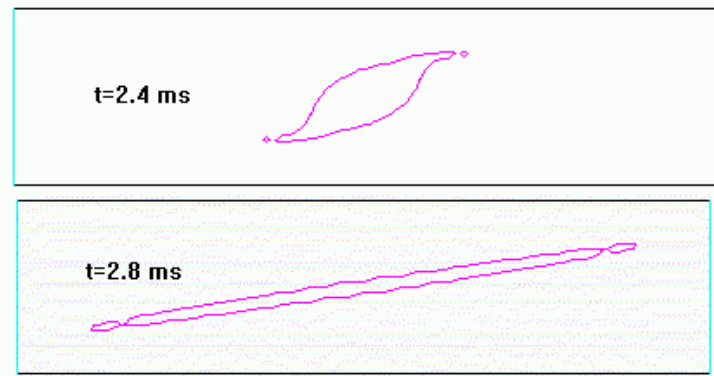

Fig. 7 Numerical evolution of rupturing behaviour of a $\mathrm{Pb}$ metallic drop in shear-induced flow. $\lambda=1$, rapid profile of initial two-sided shear rate (upper, $\mathrm{Ca}=1.70$, case6) and linear profile of initial shear rate (lower, $\mathrm{Ca}=1.17$, case 7 )
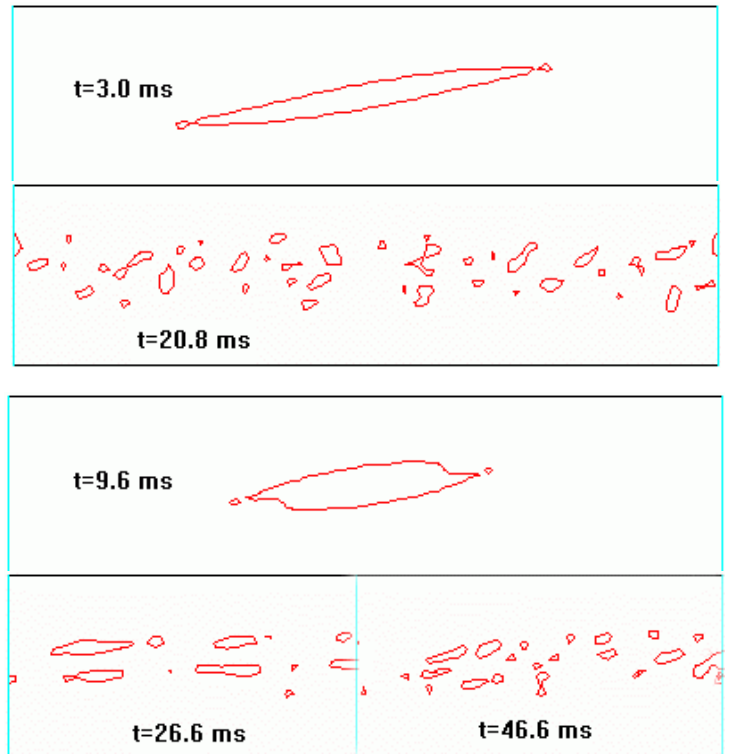

Fig.8 Results of a $\mathrm{Pb}$ metallic drop broken up into droplets by pure shear rate (nil profile of initial shear rate) at the time when the first daughter droplet was formed and complete mixing. Grid $128 \times 32$, domain $16 \times 4$, $\mathrm{Ca}=2.5$. Case 8 (upper) is for $\lambda=1$, case 9 (lower) is for $\lambda=0.5$. The breakup of a $\mathrm{Pb}$ metallic drop is easier in high viscosity flow than in a low viscosity flow 
In the rheomixing process, materials are in constant turbulent flow movement during the shearing time. The effects of turbulence and dynamic flow are shown in Fig. 9. The results show that drop breakup is much faster in dynamic shear turbulent flow than in pure shear turbulent flow. The distribution of droplets in the domain is more homogenous than in case 8 and case 9 in Fig. 8. The droplet size is also more uniform than in case 8 and case 9 . The shapes of droplets in turbulent flow and low viscosity ratio are much closer to spherical.

The coalescence of droplets occurs in an equal viscosity flow (Fig. 10) during the mixing stage, however, this did not appear in case 11 with high viscosity of matrix phase (Fig. 11). The shape of droplets in case 11 is close to spherical, as occurred in case 9 with lower viscosity ratio.

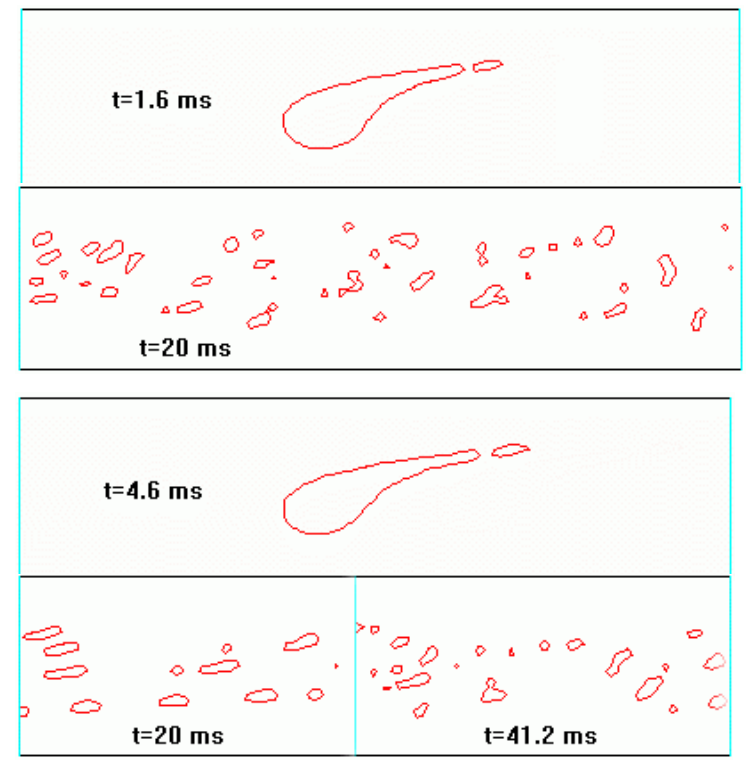

Fig.9 Results of a $\mathrm{Pb}$ metallic drop broken up in turbulent flow at the time when first daughter droplet was formed and complete mixing. Grid $128 \times 32$, domain $16 \times 4$. $\mathrm{Ca}=3.2$, case 10 (upper) is for $\lambda=1$, case 11 (lower) is for $\lambda=0.5$.

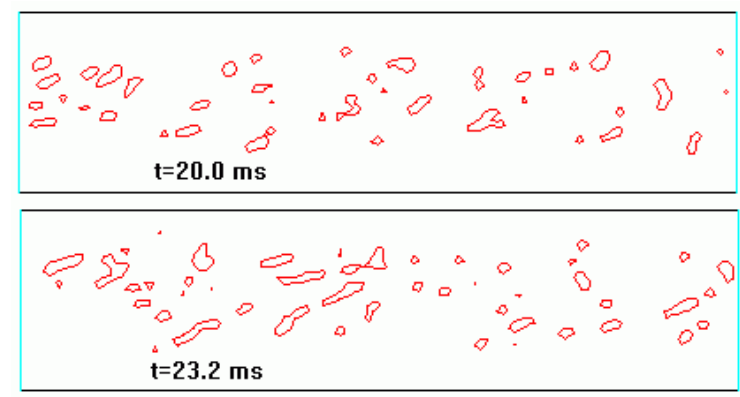

Fig.10 Illustrations of coalescence occurred in case $10, \lambda=1$. An equal viscosity leads to coalescence of $\mathrm{Pb}$ metallic droplets at the mixing stage more easily than low viscosity ratio. 


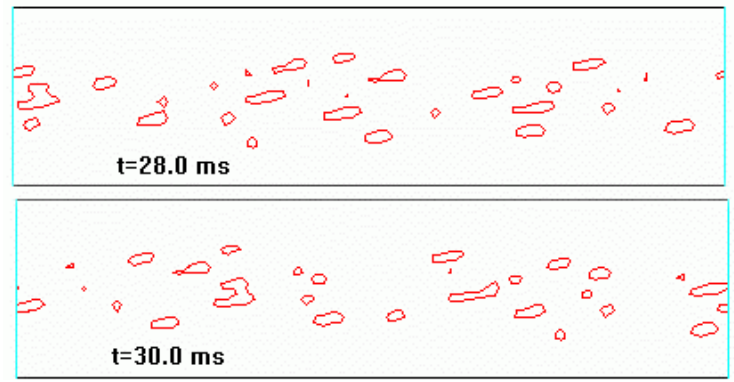

Fig.11 Illustrations of coalescence occurred in case $11, \lambda=0.5$. With high viscosity of matrix phase, these are less coalescence in the mixing stage and more spherical shape of droplets.

\subsection{Effect of shearing time in rupturing and mixing process}

Table 1 summarises the shearing time needed in each case. The shearing time to forming the first daughter drop and reaching full breakup of a parent drop is different in each case. The shearing time in the one-sided shear flow (case 4) is longer than for two-sided shear flow (case 5 and case 6 ). The low $\lambda$ value (high viscosity of the matrix phase, case 9) needs much more time than an equal viscosity flow (case 8) to reach full breakup. Turbulent flow (case 10) leads to the forming of the first daughter drop earlier than laminar flow. The low $\lambda$ value turbulent flow (case 11) also needs more time than an equal viscosity turbulent flow (case 10) to reach the full breakup.

Table 1

Comparison of shearing times for forming first daughter drop and for reaching full breakup of parent drop

\begin{tabular}{lrrrrrrrrrrc}
\hline Case No. & $\mathbf{1}$ & $\mathbf{2}$ & $\mathbf{3}$ & $\mathbf{4}$ & $\mathbf{5}$ & $\mathbf{6}$ & $\mathbf{7}$ & $\mathbf{8}$ & $\mathbf{9}$ & $\mathbf{1 0}$ & $\mathbf{1 1}$ \\
$\begin{array}{l}\text { Shearing time for } \\
\text { forming first daughter } \\
\text { drop (ms) }\end{array}$ & 4.6 & 3.2 & 100 & 2.8 & 2.2 & 2.2 & 2.8 & 3.0 & 9.6 & 1.6 & 4.6 \\
$\begin{array}{l}\text { Shearing time for } \\
\text { reaching full breakup } \\
\text { of parent drop (ms) }\end{array}$ & --- & --- & --- & 11.8 & 6.0 & 6.6 & 6.0 & 6.8 & 22 & 6.0 & 11.8 \\
\hline
\end{tabular}

After full breakup of a parent drop, droplets are in a mixing stage with further refinement and coalescence. The main concerns in the rheomixing process are maximum size of daughter droplet, uniformity of size and homogeneity of dispersion. In order to take into account these features in a qualitative discussion, we define a maximum scale factor $K$ for measuring the size of daughter droplets. 
The maximum scale factor of droplets $K_{\max }$ is defined as the ratio of the maximum size of daughter droplet to the diameter of initial parent drop at each shearing time level:

$$
K_{\max }=\frac{\max \text { size of daughter droplet }}{\text { diameter of parent drop }}=\frac{\max L_{d d}}{D_{d}}
$$

The maximum scale factor of daughter droplet $K_{\max }$ varies with shearing time, as shown in Fig.12, and generally decreases with increasing shearing time in all cases. Refinement and coalescence occur simultaneously. $K_{\max }$ is much larger in an equal viscosity system than in a low viscosity ratio system.

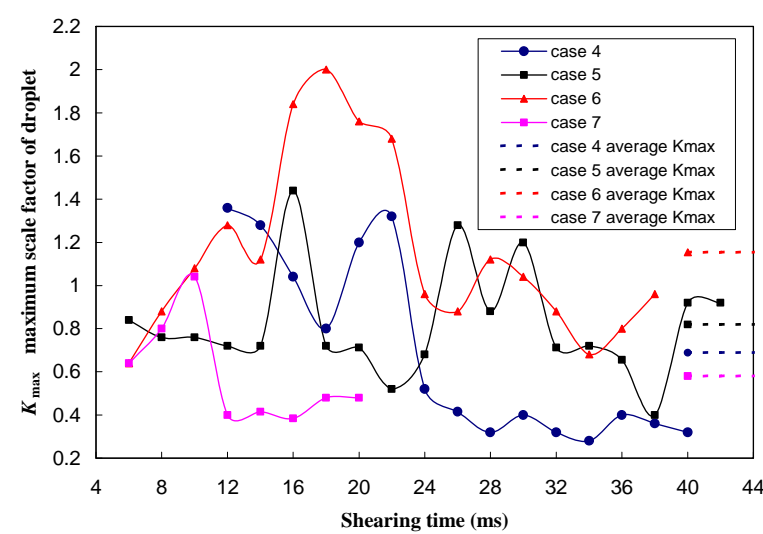

Fig.12 Comparison of maximum size of daughter droplet during shearing time for cases 4 to 7 . After fully breakup of parent drop, daughter droplets are in mixing stage with further refinement and coalescence simultaneously.

\subsection{Comparison with experimental results}

The numerical evolution of droplet dispersion is compared with an experimental image in Fig.13. It is observed that numerical results show good qualitative agreement with the experimental image [55]. The $\mathrm{Zn}-\mathrm{Pb}$ binary alloy was fed into the TSE at a temperature $550{ }^{\circ} \mathrm{C}$, the shearing temperature was set at $423{ }^{\circ} \mathrm{C}$, the rotational speed of TSE was $800 \mathrm{rpm}$. The numerical simulation is focused on the hydrodynamic behaviour of immiscible alloys in isothermal condition. Comparing the computational graph with the experimental image, the finest droplets in the right upper figure of Fig.13 are missing in the computation. In fact, in order to capture a $20 \mu \mathrm{m}$ droplet, the domain grid would need to increase hundreds times. Fortunately, the main concern in rheomixing is the maximum droplet size. Comparing the droplet distribution above $32 \mu \mathrm{m}$ of diameter, the quantitative difference is shown in the lower curve of Fig. 13. For a detailed image analysis, a shape factor $K_{\mathrm{k}}$ is defined as $4 \pi$ times the ratio of the droplet area to perimeter squared: 


$$
K_{k}=4 \pi \frac{\text { droplet area }}{(\text { droplet perimeter })^{2}}
$$

A perfect circle has a shape factor of 1 , and a line has a shape factor approaching 0 . It is noted that the maximum shape factor $K_{\mathrm{k}}$ is 1.571 for both experiment and simulation. This probably means that the maximum droplet sizes are comparable between experiment and simulation. The present results of numerical simulation show that further numerical predictions can be explored to provide a deeper insight into the microstructure mechanism of the rheomixing process.

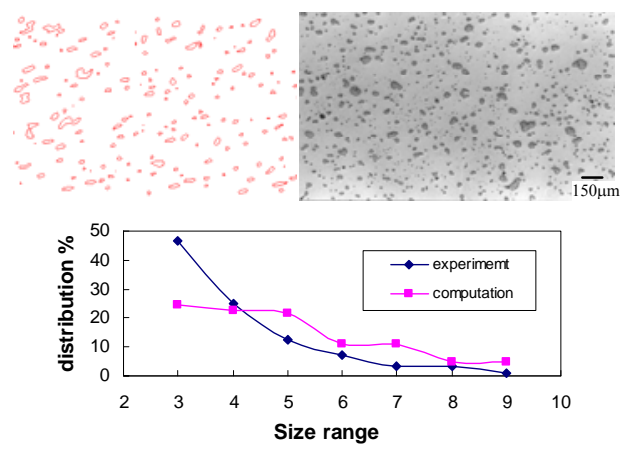

Fig.13 Comparison of the morphology of droplet dispersion, experimental image (right) [55] and numerical simulation (left) using similarity principle approach. Comparison of the droplet distribution at size range above $32 \mu \mathrm{m}$ of diameter (lower chart)

\subsection{Suggestions for optimisation of rheomixing process}

The numerical analysis presented in this paper aimed at improving our understanding of the fundamental mechanisms of the mixing of immiscible $\mathrm{Zn}-\mathrm{Pb}$ metallic alloys in shear-induced flow, which is the main flow feature of twin-screw rheomixing process. The results basically reveal that a proper control of the rheomixing process is very important since immiscible $\mathrm{Zn}-\mathrm{Pb}$ alloys have different hydrodynamic behaviours for different shearing times, shearing approaches and viscosity ratio of the alloy system. Some suggestions for optimisation of the rheomixing process are as follows:

1. Stirring the $\mathrm{Zn}-\mathrm{Pb}$ alloy at a higher shear rate in the phase separation stage before pouring it into the TSE will shorten shearing time. However, the shear rate needs to be sufficiently high to allow the entire minority phase to form large drops.

2. Shearing $\mathrm{Zn}-\mathrm{Pb}$ alloy in an equal viscosity system leads to fine daughter droplets and short shearing times, and controlling operating temperature above the monotectic temperature leads to the appearance of both phases for easier droplet breakup. 
3. Increasing the viscosity of the $\mathrm{Zn}$ phase results in more spherical droplets and avoids further coalescence.

4. Imposing turbulence in $\mathrm{Zn}-\mathrm{Pb}$ alloy system results in finer daughter droplets, uniform-sized droplets and homogeneous dispersion, as well as shorten shearing time. However, this can also cause coalescence, so flow should be kept laminar by controlling the temperature to increase the viscosity of the $\mathrm{Zn}$ phase at the certain operating point.

5. Optimal shearing approach: to start shearing the $\mathrm{Zn}-\mathrm{Pb}$ alloy above $\mathrm{T}_{\mathrm{m}}$ with enhanced turbulent flow in order to quickly obtain fine droplets, then shear it near or below $\mathrm{T}_{\mathrm{m}}$ to obtain low viscosity ratio so that droplets are close to spherical shape.

6. Twin-screw configuration is very important, since enhanced turbulent flow can be generated by assembling suitable numbers of left handed screw, right handed screw and mixing block, as well as a reasonable arrangement along the axis of twin-screw, as it has been observed experimentally that velocity fields in a twin-screw extruder are significantly affected by the structure of the screw [20].

\section{Conclusion}

This paper presented a numerical analysis of the hydrodynamic behaviour of immiscible metallic alloys in shearing mixing processes for various flow configurations, which reflect the fundamental flow features of twin-screw rheomixing process. The numerical modelling is conducted as a $2 \mathrm{D}$ periodic isothermal problem through the VOF method with PLIC scheme, AMG approach and $k-\varepsilon$ turbulence model. This paper shows that the numerical method was able to simulate the hydrodynamic behaviour of an immiscible $\mathrm{Zn}-\mathrm{Pb}$ binary alloy in shear-induced mixing processes. The rupturing, interaction and dispersion of droplets are strongly influenced by the shearing forces, viscosity ratio, turbulence, and shearing time. Since the solidified microstructure of cast immiscible alloys is strongly influenced by the hydrodynamic behaviour within the melt state, the numerical simulation of the essential microscopic mechanisms of a prototypical rheomixing process is useful for the optimisation of the rheomixing process. Possible improvements for the rheomixing process have been suggested.

\section{Acknowledgements}

We acknowledge support from EPSRC grant GN/N14033, Glacier Vanderville Ltd, Ford Motor Co. Ltd, Prism Ltd, and Qinetiq Ltd. We are also grateful to researchers around us for the helpful discussions on the operating conditions of rheomixing process and experimental images. 


\section{References}

[1] Ratke L, Diefenbach S. Liquid immiscible alloys. Materials Science and Engineering 1995; RI5:263-347.

[2] Zhao JZ, Drees S, Ratke L. Strip casting of Al-Pb alloys - a numerical analysis. Materials Science and Engineering A 2000;282:262-269.

[3] Fan Z, Ji S, J Zhang. Processing of immiscible metallic alloys by rheomixing process. Materials Science and Technology 2001; 17: 838-842.

[4] Ji S, Z Fan, Bevis MJ. Semi-solid processing of engineering alloys by a twin-screw rheomoulding process. Materials Science and Engineering A 2001;299:210-217.

[5] Cheremisinoff NP, Polymer Mixing and Extrusion Technology. New York: Marcel Dekker; 1987.

[6] Rauwendaal C. Mixing in Polymer Processing. New York: Marcel Dekker; 1991.

[7] Andersen PG. In: I. Manas-Zloczower and Z. Tadmor, Editors, Mixing and Compounding of Polymers, 679-705. New York: Hanser Publishers; 1994.

[8] Carneiro OS, Caldeira G, Covas JA. Flow patterns in twin-screw extruders. Journal of Materials Processing Technology 1999; 92-93: 309-315.

[9] White JL. Twin Screw Extrusion. New York: Hanser Publishers;1991; 254, Fig.12.2-1, original from R. Erdmenger. Chem Eng Technol 1964;36: 175.

[10] S Bakalis. Karwe MV. Velocity distributions and volume flow rates in the nip and translation regions of a co-rotating, self-wiping, twin-screw extruder. Journal of Food Engineering 2002; 51:273-282.

[11] Avalosse T. Twin screw extruder and mixing tank. presentation of Polyflow s.a., Louvain-la-Neuve, Belgium, 2000.

[12] Meijer, HEH, Janssen, JMH. In: In: I. Manas-Zloczower and Z. Tadmor, Editors, Mixing and Compounding of Polymers. New York: Hanser Publishers; 1994.

[13] Wilson MP, Al-Malaika S, Generalis SC, Bruce DP. Flow field analysis of some 3D mixing element regions within a closely intermeshing, co-rotating twin-screw extruder. AIChE Journal world congress of Chem Eng 1996.

[14] Avalosse Th, Marchal Th, Rubin Y. Analysis of mixing in co-rotating twin-screw extruders. Presented at AIChE Meeting Dallas, TX, 1999.

[15] Potente H, Flecke J. Analysis and modelling of the residence time distribution in intermeshing co-rotating twin-screw extruder based on finite element simulation. In: Proc. of the ANTEC'97 conference, Brookfield CT, 1.1 (1997) 110-114, Society of Plastics Engineers, Toronto, Canada, 1996.

[16] Avalosse Th, Rubin Y, L Fondin. Non-isothermal modeling of co-rotating and contra-rotating twin screw extruders. In: Proc. of the ANTEC 2000 conference Brookfield CT, Vol XLVI, p19-23, Society of Plastics Engineers, Orlando, FL, USA, 2000.

[17] Avalosse Th, Rubin Y. Analysis of mixing in co-rotating twin screw extruders through numerical simulation. In: Proc. of the PPS-15 conference, 's-Hertogenbosch, The Netherlands, 1999.

[18] van Zuilichem DJ, Kuiper E, Stoplp W, Jager T. Mixing effects of constituting elements of mixing screws in single and twin screw extruders. Power Technology 1999; 106: 147-159.

[19] Bourry D, Favis BD. Morphology development in a polyethylene / polystyrene binary blend during twin-screw extrusion. Polymer 1998; 39:1851-1856.

[20] Bakalis S, Karwe MV. Velocity field in a twin-screw extruder. Int. J. Food Science and Technology 1997; 32: $241-253$.

[21] Taylor GI. The viscosity of a fluid containing small drops of another fluid. In: Proc. R. Soc. London, Ser.A138: 41, 1932.

[22] Taylor GI. The formation of emulsions in definable fields of flow. In: Proc. R. Soc. London, Ser.A146: $501,1934$.

[23] Stone HA. Dynamics of drop deformation and breakup in viscous fluids. Annu Rev Fluid Mech 1994; 26:65-102.

[24] Bentley BJ, Leal LG. An experimental investigation of drop deformation and breakup in steady, two-dimensional linear flows. J Fluid Mech 1986; 167:241-283.

[25] Kennedy MR, Pozrikidis C, Skalak R. Motion and deformation of liquid drops, and the rheology of dilute emulsion in simple shear flow. Computers Fluids 1994; 23: 251-278.

[26] Siegel M. Influence of surfactant on rounded and pointed bubbles in two-dimensional Stokes flow. SIAM J Appl Math 1998; 59.

[27] Loewenberg M, Hinch EJ. Numerical simulation of a concentrated emulsion in shear flow. J Fluid Mech 1996; 321: 395-419.

[28] Scardovelli R, Zaleski S. Direct numerical simulation of free surface and interfacial flow. Annu Rev Fluid Mech 1999; 31: 567-603.

[29] Briscoe BJ, Lawrence CJ, Mietus WGP. A review of immiscible fluid mixing. Advances in Colloid and Interface Science 1999; 81: 1-17. 
[30] Hirt CW, Nichols BD. Volume of fluid VOF for the dynamics of free boundaries. J Comput Phys 1981; 39: 201-225.

[31] Youngs DL, Time-dependent multi-material flow with large fluid distortion. In: Morton KW, Baines MJ, editors. Numerical Methods for Fluid Dynamics, New York: Academic;1982; 273-285.

[32] Stüben K. A review of algebraic multigrid. Journal of Computational and Applied Mathematics 2001; 128: 281-309.

[33] Launder BE, Spalding DB. The numerical computation of turbulent flows. Comput Meth App Mech Engng 1974; 3 : 267-289.

[34] Tang H, Wrobel LC, Fan Z. Numerical evaluation of immiscible metallic Zn-Pb binary alloys in shear-induced turbulent flow. Materials Science and Engineering A. special issue for $1^{\text {st }}$ international Conference on Mulltiscale Material Modelling, London, 2002.

[35] Rauwendaal C. Polymer Extrusion. $3^{\text {rd }}$ ed. New York: Hanser Publisher; 1985.

[36] Sundararaj,U, Dori Y, Macosko CW. Sheet formation in immiscible polymer blends: model experiments on initial blend morphology. Polymer 1995; 36:1957-1968.

[37] Fan Z, Bevis MJ, Ji S, UK Patent, Application No 9922696.3, 1999.

[38] Iida T, Guthrie RIL. The Physical Properties of Liquid Metals. New York: Oxford University Press; 1988.

[39] R Hultgren RL, Orr, Anderson PD, Kelley KK. Selected Values of the Thermoldynamic Properties of Binary Alloys. Metals Park, OH: ASM;1973.

[40] Unverdi SO, G Tryggyason. A front-tracking method for viscous, incompressible, multi-fluid flows. J Comput Phys 1999; 100: 25.

[41] Cristini V, Blawzdziewicz J, Loewenberg M. Drop breakup in three-dimensional viscous flows. Physics Fluids 1998; 10:1781-1783.

[42] Rider WJ, Kothe DB. Reconstructing volume tracking. J Comput Phys 1998; 141: 112-152.

[43] Lafaurie B, Nardone C, Scardovelli R, Zaleski S, Zanetti G. Modeling merging and fragmentation in multiphase flows with SURFER. J Comput Phys 1994; 113: 134.

[44] Nichols BD, Hirt CW, Hotchkiss RS. SOLA-VOF: A solution algorithm for transient fluid flow with multiple free boundaries. Los Alamos Scientific Laboratory Report LA-8355;1980.

[45] Kothe DB, Mjolsness RC, Torrey MD. RIPPLE: A computer program for incompressible flows with free surfaces. Los Alamos National Laboratory Report No. LA-12007-MS;1991.

[46] Zaleski S, Li J, Succi S. Two-dimensional Navier-Stokes simulation of deformation and breakup of liquid patches. Phys Rev Lett 1995; 75: 244.

[47] Osherand S, Sethian JA. Fronts propagating with curvature-dependent speed: algorithms based on Hamilton-Jacobi formulations. J Comput Phys 1988; 79:12.

[48] Brackbill JU, Kothe DB, Zemach C. A continuum method for modeling surface tension. J Comput Phys 1992; 100:335-354.

[49] Tang H, Barton IE, Fan Z. Numerical experimental investigation of particle interaction in the turbulent flow through a 10:1:10 constriction, submitted to Powder Technology 2002.

[50] Issa RI, Gosman AD, Watkins AP. The computation of compressible and incompressible recirculating flows by a noniterative implicit scheme. J Comput Phys 1991; 93: 388-410.

[51] Li J, Renardy YY, Renardy M. A numerical steady of periodic distributions on two-layer Couette flow. Physics of Fluids 1998; 10: 3056-3071.

[52] Joseph DD, Nguyen K, Beavers G. Non-uniqueness and stability of the configuration of flow of immiscible fluids with different viscosities. J Fluid Mech 1984; 141: 319-345.

[53] Barthelet P, Charru F, Benjamin-Feir and Eckhaus instabilities with Galilean invariance: the case of interfacial waves in viscous shear flows. Eur J Mech B/Fluids 1998; 17: 1-18.

[54] Charru F, Barthelet P. Secondary instabilities of interfacial waves due to coupling with a long wave in a two-layer Couette flow. Physica D 1999; 125: 311-324.

[55] Fang X, Fan Z. Ji S, Hu Y. Processing of immiscible alloys by a twin-screw rheomixing process. In: Tsutsui Y, Kiuchi M, Ichikawa K. ed. Proceeding of the $7^{\text {th }}$ Advanced Semisolid Processing of Alloys and Composites, Tsukuba 25-27 September 2002, Japan: NIAIST, JSTP, 695-700. 